\title{
Lo técnico y lo político en la política social.
}

\section{Reflexiones a partir de} la experiencia de una cooperativa apoyada por políticas de promoción del trabajo asociativo y autogestionado en Argentina

The technical and the political in social policy. Reflections from the experience of a cooperative supported by policies to promote associative and self-managed work in Argentina

\section{Malena Victoria Hopp}

Licenciada en Trabajo Social

(Universidad Nacional de Buenos Aires)

Especialista en Planificación y Gestión de Políticas Sociales

(Universidad Nacional de Buenos Aires)

Magister en Políticas Sociales

(Universidad Nacional de Buenos Aires)

Doctora en Ciencias Sociales

(Universidad Nacional de Buenos Aires)

Investigadora adjunta (CONICET)

(Instituto de Investigaciones Gino Germani)

Investigadora del Centro Cultural de la Cooperación

Docente en la carrera de Trabajo Social

(Universidad Nacional de Buenos Aires)

Correo: malenahopp@yahoo.com.ar 


\section{Resumen}

El objetivo de este artículo es analizar las representaciones acerca de lo técnico y lo político que construyen distintes actores vinculades con las políticas sociales de promoción del trabajo asociativo autogestionado, implementadas en Argentina entre 2003 y 2011. Para ello utilizamos una metodología cualitativa en base a un trabajo de campo etnográfico. A partir del análisis de la experiencia de una cooperativa de trabajo apoyada por varias de estas políticas, mostramos el modo en que estas representaciones sobre lo técnico y lo político, se ponen en juego en las prácticas cotidianas y moldean los límites y las condiciones de la participación y distribución de tareas entre "profesionales", "militantes" y "cooperativistas". El análisis realizado permite pensar el Trabajo Social, sus fortalezas y desafíos, a partir del reconocimiento de la centralidad de la escala cotidiana de la política social, entendida como un momento determinante de su institucionalidad y capacidad de intervención en las condiciones de vida y de integración social (Grassi, 2014).

Palabras clave

Política social, Trabajo Social, Intervención social, Trabajo asociativo autogestionado.

\section{Abstract}

The objective of this article is to analyze the representations about the technical and the political that are constructed by different actors linked to the social policies to promote self-managed associative work, implemented in Argentina between 2003 and 2011. We use a qualitative methodology based on an ethnographic field work, located in a cooperative supported by several of these social programs. The article shows the way in which these representations about the technical and the political are put into play in everyday life practices and shape the limits and conditions of the participation and distribution of tasks and responsibilities among "professionals", "militants" and "cooperative members". The analysis opens up new questions about Social Work, its strengths and challenges, from the recognition of the importance of everyday social politics, understood as a determining moment of its institutionality and capacity for intervention in the life conditions and social integration (Grassi, 2014).

\section{Keywords}

Social policy, Social Work, Social intervention, Self-managed associative work. 
Las reflexiones que presentaremos surgen a partir de la investigación que tuvo como resultado el libro El trabajo: ¿medio de integración o recurso de la asistencia? (Hopp, 2021). El problema que abordamos allí fue en qué medida las experiencias laborales apoyadas por las políticas sociales de promoción del trabajo asociativo y autogestionado, implementadas en Argentina entre los años 2003 y 2011, alcanzan el objetivo que se proponen de integrar a quienes en ellas participan a las condiciones socialmente aceptables y aceptadas de la vida social. En este artículo nos proponemos volver sobre una cuestión que se presentó como un emergente del trabajo de campo realizado en aquel entonces y que concluida dicha investigación, abre nuevas preguntas y nos permite pensar el Trabajo Social, sus fortalezas y algunos desafíos, a partir del reconocimiento de la centralidad de la escala cotidiana de la política social, entendida como un momento determinante de su institucionalidad y capacidad de intervención en las condiciones de vida y de integración social (Grassi, 2014).

En el desarrollo del trabajo de campo realizado, observamos recurrentemente representaciones que marcaban una oposición entre lo "técnico" y lo "político", tanto al interior de las organizaciones sociales y cooperativas, como en las agencias estatales que participan de la implementación de estos programas. Estos términos que se planteaban como opuestos, suponían también distintas lógicas de acción y formas de concepción de la política social y de sus propósitos. En este marco, el objetivo de este artículo es analizar las representaciones acerca de lo técnico y lo político que construyen distintes actores vinculades con las políticas sociales de promoción del trabajo asociativo autogestionado, implementadas en Argentina en el periodo 2003-2011.

Para ello, primero presentaremos la perspectiva teórico-metodológica con la que abordamos las políticas sociales, en particular, los programas de promoción del trabajo asociativo autogestionado. Luego, a partir del análisis de la experiencia de una cooperativa de trabajo que fue apoyada por varios de estos programas, intentaremos mostrar el modo en que estas representaciones y valoraciones sobre lo técnico y lo político, se ponen en juego en las prácticas cotidianas y moldean los límites y las condiciones de la participación y distribución de tareas y responsabilidades entre "profesionales", "militantes" y "cooperativistas". Por último, plantearemos algunas reflexiones que nos permiten pensar el ejercicio profesional del Trabajo 
Social, a partir de comprender la centralidad de la escala cotidiana de la política social en el acceso a derechos y la consecución de los objetivos propuestos por las políticas diseñadas desde el nivel central del Estado.

\section{Abordaje teórico-metodológico}

Como mencionamos, el problema que abordamos en la investigación realizada (Hopp, 2021) fue en qué medida las experiencias de trabajo asociativo y autogestionado promovidas por las políticas sociales, alcanzan el objetivo que se proponen de integrar social y económicamente a personas y grupos que en ellas participan. El objeto se delimitó por la vinculación entre las políticas sociales, el trabajo y las formas de integración social que a partir de esta relación se despliegan. Para construir la estrategia metodológica, partimos de considerar las políticas sociales como procesos que articulan el nivel estructural de los procesos político-sociales con la dimensión de la vida cotidiana en la que las personas despliegan sus prácticas. Las distintas instancias que conforman las políticas sociales (diagnóstico, planificación, diseño, instrumentación, evaluación, etc.) las definimos como momentos de la formación o realización de la política (Minteguiaga, 2003).

Esta noción de momentos se opone a la idea de fases o etapas ${ }^{1}$, que supone una escisión entre la planificación y el diseño de la política y su instrumentación y que le atribuye a cada una de estas etapas una lógica de funcionamiento diferencial, en la cual se considera la primera etapa del diseño como una fase política y, la segunda de instrumentación, como una instancia técnico-administrativa. Desde esta perspectiva, el trabajo social se ubicaría en esta segunda instancia considerada "técnica", aunque como veremos a partir del caso que analizaremos, esta oposición no es tal, ya que en todos los momentos se toman decisiones "técnicas" y "políticas" que resultan determinantes para la implementación de los programas y la consecución de los objetivos propuestos. Esta distinción de momentos es productiva para el análisis de las políticas sociales en la medida en que no los concibamos como instancias autónomas, sino como procesos interde- 
pendientes. Tal distinción, tampoco implica considerar alguna de las instancias como más relevante o decisiva que las otras, ya que en todas ellas se realizan actividades y procedimientos de definición, decisión y ejecución, en los que se ponen en juego y se disputan intereses y sentidos. Por ello, debemos mirar las políticas en su trayectoria global (Danani, 1996).

Como señala Shore, en contraste con la idea de una "pulcritud abstracta" (2010:29) de la formulación de políticas públicas, es necesario mostrar la complejidad y el desorden de dicho proceso, las formas en que sus lineamientos son recibidos y disputados por les agentes involucrades y sus destinataries. Desde esta perspectiva también podemos discutir el enfoque que analiza las políticas públicas a partir de una división tajante entre unidades y procesos intraburocráticos y procesos que se situarían "por fuera" del Estado. Siguiendo a Trouillot (2001), afirmamos que el poder del Estado no tiene fijeza institucional y sus efectos no se producen exclusivamente en los sitios gubernamentales. La política estatal se encuentra necesariamente inscripta en procesos sociales más amplios que exceden el aparato estatal y las instituciones públicas, en los que les distintes actores no cuentan con los mismos medios y recursos para imponer la direccionalidad y el sentido de las acciones (Minteguiaga, 2003).

En el análisis de las políticas sociales y en nuestra investigación en particular, cobró centralidad la dimensión cotidiana del proceso de desarrollo de los programas de promoción del trabajo asociativo y autogestionado y la trama de relaciones que se tejen en las interacciones entre agencias estatales, organizaciones, referentes polítiques o territoriales y destinataries de los mismos. Es en ese espacio de la vida diaria en donde se (re)producen, transforman y disputan los sentidos sobre el trabajo y los recursos que ofrece la política social y se ponen en juego las distintas representaciones sobre lo técnico y lo político. Es allí también donde se despliega el ejercicio profesional del Trabajo Social, que entendemos como una práctica que implica tanto la teoría y la producción de conocimiento sobre los problemas en los que es llamado a intervenir, como el despliegue de instrumentos técnicos, que se vinculan, a su vez, con determinado posicionamiento ético-político. En este ejercicio, les trabajadores sociales no somos meros operadores o ejecutores de las políticas sociales. Como plantea Aquin (2002) participamos en tres momentos que forman parte del objeto de la disciplina: en la lucha por validar una necesidad como problema social; en la interpretación 
de la misma como un problema político o no político, es decir en la disputa por el modo en que los problemas son definidos y colocados (o no) en la agenda pública, y en la asignación de recursos para su abordaje, que solo es posible si una necesidad es considerada como un problema político y que dependerá también del sentido e interpretación construidas.

Como señala Grassi (2014), la normatividad de las políticas sociales adquiere materialidad en cada microcosmos, es allí en donde se despliegan los contenidos de la norma, así como también los modos de interpretación y su manipulación por quienes participan en las diferentes agencias y ámbitos de aplicación en los distintos niveles del Estado (central, provincial, local), y en las diferentes organizaciones que llevan adelante estas políticas como entes ejecutores ${ }^{2}$. Los efectos de las políticas se producen en la conjunción de las intervenciones y recursos que proveen los programas y los modos en que les sujetes, grupos y organizaciones se apropian y transforman dichos recursos, a partir de sus experiencias de vida, su capacidad organizativa y los proyectos sociopolíticos colectivos que orientan sus prácticas (Hopp, 2015). Por eso, el análisis no puede centrarse exclusivamente en el Estado, sino que requiere un enfoque relacional (Bourdieu y Wacquant, 1995; Hintze y Deux, 2007) que se sitúe en las interacciones entre les distintes agentes durante el proceso de implementación de los programas.

El abordaje del problema planteado y la comprensión de las representaciones sobre lo técnico y lo político, no pueden centrarse tampoco en el plano normativo de aquello que las políticas sociales deben ser, ni orientarse a evaluar las incongruencias entre su diseño, sus objetivos y los resultados efectivamente alcanzados. Desde nuestra perspectiva de análisis, se trata de comprender el proceso de la política de promoción del trabajo asociativo autogestionado como un todo, que incluye tanto la formulación de los programas en el nivel gubernamental central, como las prácticas y relaciones entre agencias estatales y entre éstas y les destinataries y actores sociales que participan en dicho proceso. No sólo los requisitos formales, "técnicos" o burocráticos de acceso, sino los modos en que éstos funcionan y cómo se articulan, por ejemplo, con los acuerdos "políticos" o, por el contrario, po-

2 De allí la centralidad de integrar al estudio de la política social, la reflexión sobre estos ámbitos y relaciones que se despliegan en la escala cotidiana de la política y que son constituyentes del momento del encuentro de los usuarios con las prestaciones de la política (Grassi, 2014). 
nen en cuestión u obstaculizan tales acuerdos cuando la voluntad de otorgar recursos se encuentra desligada o desconsidera el proceso técnico-burocrático propio y necesario para la gestión de las políticas sociales.

\section{El desarrollo del trabajo de campo}

Nuestra estrategia metodológica se orientó a explorar las mediaciones entre las políticas y las prácticas sociales, a fin de reconstruir la compleja vinculación entre los programas y las prácticas sociales en la trama de relaciones que da forma concreta a las políticas sociales. Para ello optamos por un abordaje socio-antropológico (Guber, 2009) y el desarrollo de un trabajo de campo prolongado que incluyó la observación participante y la realización de entrevistas semiestructuradas, en el estudio de un caso en profundidad. El trabajo de campo se desarrolló entre marzo de 2009 y febrero de 2011 y se localizó en una cooperativa de trabajo, situada en la Ciudad Autónoma de Buenos Aires, cuyo nombre ficticio es La Huella ${ }^{3}$.

Se trata de una cooperativa conformada por quince trabajadores y trabajadoras, que se encontraba en funcionamiento y consolidada y que recibió apoyo de programas de promoción del trabajo asociativo y autogestionado, en particular de la línea de subsidios del Plan Manos a la Obra y de la línea de microcréditos, implementadas por el Ministerio de Desarrollo Social y del Programa Trabajo Autogestionado, dependiente del Ministerio de Trabajo, Empleo y Seguridad Social ${ }^{4}$. La cooperativa tenía una fuerte relación

3 Para resguardar la privacidad de les informantes y la confidencialidad de sus relatos modificamos el nombre de la cooperativa y de sus integrantes, a fin de garantizar el anonimato de los mismos.

4 En 2003, comenzó un proceso de reestructuración de las políticas sociales y laborales en Argentina destinado a afrontar la crisis socioeconómica y los altísimos niveles de pobreza y desocupación que se registraban en ese entonces, mediante la generación de trabajo genuino. Desde el discurso oficial la nueva estrategia recolocaba al trabajo en el centro de la política pública, marcando una clara diferencia respecto de gobiernos anteriores que vehiculizaron la instauración del modelo neoliberal y diversas medidas de ajuste estructural que se interpretaban como las causas de estos problemas que debían ser atendidos de manera urgente. La nueva política social se distinguía y denunciaba la focalización y el asistencialismo de los planes que habían sido destinados a atenderlos previamente. En este contexto, desde el Ministerio de Desarrollo Social se creó el Plan Nacional de Desarrollo Local y Economía Social "Manos a la Obra", cuyo propósito fue constituir un sistema de apoyo a las experiencias de desarrollo socioeconómico local, orientado a sectores de bajos recursos. A partir de este Plan, la promoción del trabajo asociativo y autogestionado en el marco de la Economía Social tomó un rol relevante en el abordaje de los problemas de desempleo y pobreza. Entre 2003 y 2011, se produjo un proceso de ampliación de esta línea de política social que formó parte de las disputas político-culturales por imponer un nuevo sentido del trabajo en los procesos de integración social y la orientación de las intervenciones sociales del Estado destinadas a su promoción y desarrollo (Hopp, 2021). Esta línea que continuó y se consolidó durante todo el ciclo 
con las agencias gubernamentales que implementaban estos programas y parte de sus estrategias de sostenimiento se basaban en la búsqueda y gestión de recursos provenientes de los mismos. También estaba estrechamente vinculada con otras experiencias y organizaciones cooperativas y asociativas que podemos considerar como parte de la Economía Social ${ }^{5}$.

Considerando la cuestión del acceso al campo (Rockwell, 2009), el contacto con La Huella fue a través de una organización de cooperativas con la que teníamos una relación previa y en la que habíamos participado en algunos espacios de capacitación y apoyo para les integrantes de la misma. En la planificación inicial, pensamos que el trabajo de campo se iba a localizar exclusivamente en el espacio laboral de la cooperativa, pero al realizar las primeras visitas, observamos que participaban activamente en la organización social a través de la cual habíamos hecho el primer contacto. Por ello a partir del principio metodológico que propone Latour (2008) comenzamos a seguir a les actores 6 , a fin de captar las relaciones que ellos mismos establecen y no delimitarlas de antemano. Así realizamos observaciones participantes en distintas reuniones y asambleas, situando el trabajo de campo en diferentes espacios. Esto posibilitó también un acercamiento a otras cooperativas que nos contaron sus experiencias en relación con las políticas de promoción del trabajo asociativo autogestionado que estábamos estudiando. Además, a partir del vínculo de la cooperativa La Huella con esta Organización, pudimos analizar algunos aspectos de cómo juega la

kirchnerista hasta 2015, incluyó diversos instrumentos, programas y nuevas instituciones de promoción, regulación y protección. Entre ellos, subsidios para la compra de herramientas e insumos de trabajo, microcréditos -de la mano de la sanción de la Ley 26.117-, la generación de cooperativas de trabajo para la realización de obras públicas en el marco de distintos programas de inclusión social, entre ellos, el Programa Ingreso Social con Trabajo Argentina Trabaja y Ellas Hacen, capacitación, asistencia técnica, la creación del Registro Nacional de Efectores de la Economía Social y el monotributo social para apoyar la formalización y fortalecer la protección del trabajo autogestionado, entre otras.

5 El criterio principal de selección de la unidad de estudio fue el carácter asociativo y la intensidad de las relaciones que las unidades productivas estudiadas tuvieran con los programas y agencias de promoción del trabajo asociativo y autogestionado.

Otro de los criterios, fue considerar que las mismas estuvieran en funcionamiento, es decir que se tratara de experiencias consolidadas que permitieran observar las características y tensiones propias de estas formas laborales, tratando de diferenciarlas de problemas que tuvieran que ver con el inicio de la actividad económica.

6 Para este autor se deben "seguir" todas las relaciones que despliegan les actores, también las de los "productos" u "objetos". En la investigación realizada (Hopp, 2021) observamos algunas de ellas, como por ejemplo el modo en que circula lo que estas cooperativas producen. 
relación con otres actores en el acceso a los recursos estatales y conocer las formas en que la organización social puede facilitar el acercamiento a los programas, a través de la demanda colectiva o el contacto con funcionaries y referentes polítiques.

Siguiendo las vinculaciones de les integrantes de la Cooperativa La Huella y sus prácticas cotidianas, realizamos observaciones en distintas dependencias estatales. Desde una "entrada" distinta, acompañamos a sus integrantes y colaboramos haciendo algunos trámites vinculados con la gestión de recursos estatales. Esto nos permitió estar en las agencias públicas del lado de les usuaries y nos ofreció una perspectiva diferente a aquella que puede captarse desde el lugar institucional ocupado habitualmente por el Trabajo Social.

\section{Lo técnico y lo político en las políticas de promoción del trabajo asociativo y autogestionado}

En este apartado, problematizaremos las representaciones que construyen distintes agentes sobre lo técnico y lo político en la política social de promoción del trabajo asociativo y autogestionado. Nos interesa indagar en estas representaciones en tanto productoras de sentidos y organizadoras de las prácticas sociales. Las representaciones son instrumentos de aprehensión de la realidad que son construidos desde una mirada y una posición específica, pero se perciben como lo real y construyen lo visible (Penna, 1992). En ese sentido, buscamos comprender el modo en que estas construcciones realizadas a partir de un proceso de selección de ciertos atributos que reducen y simplifican la complejidad del mundo social contribuyen a su naturalización. Al mismo tiempo, se trata de categorías que son disputadas y reinterpretadas en las prácticas, situaciones y contextos específicos.

$\mathrm{Al}$ momento del desarrollo de nuestro trabajo de campo, la Organización social de la cual formaba parte la Cooperativa La Huella, era administradora de un fondo de microcrédito de un programa de la Comisión Nacional de Microcrédito (en adelante, CONAMI), del Ministerio de Desarrollo Social de la Nación. El equipo encargado de la gestión del programa estaba compuesto por tres integrantes de la Organización, uno de ellos economista, otra trabajadora social y el 
último no tenía una formación profesional específica. Todes elles percibían un ingreso como retribución por las tareas de asesoramiento y acompañamiento a quienes solicitaban los créditos, que eran cubiertos con los recursos que proveía el programa para les denominades "promotores de microcrédito”. El propio diseño del programa permitía que las principales decisiones sobre quiénes accedían a los créditos y el monto que recibían, las tomara el equipo de la Organización a cargo, aunque con ciertos límites establecidos a partir de los requisitos formales y normativos del mismo ${ }^{7}$, que sin embargo, muchas veces eran disputados, negociados o reinventados en la práctica cotidiana, a fin de atender de una manera más adecuada a las necesidades y demandas concretas de las cooperativas y emprendimientos asociativos, vinculados con la Organización ${ }^{8}$.

En esta experiencia de gestión de los microcréditos, observamos dos posiciones contrapuestas respecto del rol de la política social y los usos de los recursos provenientes de las mismas. Para algunos, la relevancia de este instrumento de financiamiento radicaba principalmente en la posibilidad de fortalecer y asegurar la viabilidad de los proyectos productivos de quienes solicitaban los fondos. Por ello, se enfatizaba la importancia de brindar asistencia técnica y realizar un seguimiento de los préstamos otorgados, así como también una evaluación efectiva de las posibilidades que tenían las cooperativas de devolver el dinero recibido. Para otros, la función del microcrédito se vinculaba centralmente con la construcción de una herramienta política para favorecer el acercamiento de nuevas experiencias asociativas y autogestiona-

$7 \mathrm{Al}$ respecto, en una asamblea Fernando, referente de una cooperativa de producción de lácteos afirmaba que el microcrédito "no es un fondo importante, pero auxilia bastante" (Referente de cooperativa de producción de lácteos, 18 de diciembre de 2009). Además destacaba que la experiencia sirvió para mostrar que las organizaciones pueden administrar fondos eficaz y exitosamente.

8 Un ejemplo de ello fue que, a partir de una demanda de la Organización, luego del primer año de ejecución, lograron incrementar la cantidad de cuotas permitidas para la devolución de los créditos, lo cual permitía reducir el monto de devolución mensual y facilitar el cumplimiento de los plazos de pago por parte de quienes tomaban los créditos. Otro punto de discusión respecto de los requisitos de acceso fue el carácter individual del crédito que estipulaba el programa, que se contraponía al carácter colectivo de las experiencias de autogestión del trabajo que elles buscaban financiar. Otros procesos de disputa por el sentido de los requisitos formales en la implementación de políticas de microcrédito son analizados en Litman (2017) en términos del trabajo de apropiación creativa de los lenguajes técnicos del Estado que propone Poole (2012). 
das a la organización. Desde esta mirada todos los aspectos definidos como "técnicos", vinculados con la evaluación, el seguimiento y la rendición de cuentas a través de formularios y planillas requeridas por el Ministerio de Desarrollo Social, se interpretaban como requisitos impuestos desde el programa, desvinculados e incluso opuestos a la finalidad "política" de apoyo a les compañeres de las cooperativas y el fortalecimiento de la organización colectiva que se proponían. En este sentido, uno de los promotores del microcrédito destacaba que para él esto tuvo un impacto político muy importante, porque hubo cooperativas que se sumaron a la organización a través del microcrédito (Promotor de microcrédito, 18 de diciembre de 2009) 9 .

Estas visiones contrapuestas acerca de la política social se vinculaban con la experiencia y rol en la Organización, la formación y la trayectoria, técnico-profesional o política-militante, de quienes conformaban el equipo de gestión del programa de microcréditos. Estas trayectorias marcaban el sentido de lo "técnico" y lo "político" en sus prácticas. Desde la primera perspectiva, los procedimientos requeridos para otorgar los créditos y la evaluación de viabilidad de los proyectos eran considerados no sólo como instancias necesarias del proceso de implementación de la política social, sino que, además, al ser ejercidas por "compañeres" no funcionarían como mecanismos de control, sino como formas de apoyo y acompañamiento de los emprendimientos y cooperativas. Desde la segunda mirada, lo "técnico" era pensado como un requisito burocrático de la política social, que funcionaba como un obstáculo para el despliegue de los propios objetivos considerados como "políticos". En este marco, lo "político" es aquello que da sentido a las acciones orientadas hacia la transformación de las formas de producción y del trabajo que llevan adelante como colectivo, en contraposición a las exigencias de la intervención estatal que se conciben como obligaciones técnico-burocráticas exteriores, impuestas y carentes de utilidad.

9 Más allá de este aspecto positivo, la trabajadora social planteaba una preocupación respecto de las dificultades que se presentaban frecuentemente para devolver las cuotas del crédito a tiempo y la necesidad de reforzar el acompañamiento de los proyectos productivos. 
La distinción entre las funciones "técnicas" y "políticas" presente en las representaciones de les integrantes de La Organización, marcaba también los límites y las condiciones de la participación y la distribución de tareas y responsabilidades entre "profesionales", "militantes" y "cooperativistas". Esto se observaba en la distribución de tareas cotidianas, por ejemplo, la trabajadora social y otres profesionales se encargaban habitualmente de colaborar con las cooperativas en la elaboración de los proyectos, brindar capacitaciones y diversas cuestiones administrativas y los referentes de la Organización, intervenían en los procesos de recuperación de otras empresas, la articulación con otres actores sociales y políticos o las relaciones con funcionaries públiques para plantear sus demandas. Cabe aclarar que esta distinción entre profesionales y cooperativistas que proponemos refiere a los roles y funciones predominantes (no excluyentes) que asumía cada agente. En el caso del programa de microcrédito analizado, se vinculaba con distintos modos de entender la política social y su intervención desde el doble rol que ejercían como agentes estatales encargados de la implementación del programa y militantes de la Organización.

Al respecto, en una asamblea a la que asistimos registramos un debate acerca de si se debía continuar o no apoyando a una fábrica que había iniciado el proceso de recuperación y a la que se le estaba brindando asesoramiento jurídico, debido a un conflicto con otra organización (de otro signo político y con una mirada diferente respecto de cómo encarar la puesta en marcha de la producción) que también estaba interviniendo en la recuperación de la empresa. En el marco de esta discusión una contadora que no integraba la Organización, pero participaba habitualmente de este tipo de reuniones y colaboraba con las cooperativas realizando los balances económicos y otras cuestiones económico-contables, planteó que ella quería seguir capacitando a les integrantes de esa fábrica, más allá de los conflictos y desacuerdos políticos. Frente a su postura un cooperativista planteó indignado que para él "una cosa es la decisión del técnico individual” y otra "la decisión política” que debía tomar la Organización respecto al modo y la continuidad del apoyo a esa empresa recuperada. Luego distinguía entre quienes son "orgánicos de la Organización y pueden participar en reuniones y tomar decisiones" y "los técnicos", "si sos técnico, no 
podés participar de las reuniones”, afirmaba enfáticamente (Cooperativista, 19 de noviembre de 2009).

En esta discusión, se advierte la representación de lo técnico como aquello que se asocia a un saber individual que se encuentra por fuera y aparentemente en oposición a la Organización que se concibe como colectiva y "política". Este carácter colectivo puede entenderse como un valor que justificaría la exclusión de les llamades "técnicos" ${ }^{10}$ de las instancias de toma de decisiones, concibiéndose su intervención como la ejecución neutral de lineamientos acordados en una instancia política anterior y de mayor relevancia. Al mismo tiempo, la situación descripta deja entrever las relaciones de poder al interior de la Organización y la disputa por dar legitimidad a las decisiones que cada une sostiene, en un caso, continuar con las capacitaciones y, en el otro, desvincularse del proceso de recuperación de la fábrica, debido al desacuerdo político entre organizaciones. La interpretación de la decisión de la contadora como despolitizada e individualista que realiza el cooperativista puede entenderse también como una estrategia para desacreditar una posición opuesta a la propia ${ }^{11}$.

Por otra parte, los conflictos relevados forman parte de una discusión acerca del rol del saber técnico y de la inserción de profesionales portadores de este conocimiento especializado en las organizaciones sociales. El desarrollo de experiencias de trabajo asociativo autogestionado requiere del trabajo articulado de distintos actores sociales, y la innovación organizativa y productiva para fortalecerlas, implica la socialización de saberes de diferente tipo, así como también, la construcción de nuevos conocimientos que favorezcan estas formas de organización del trabajo. La concepción dicotómica de lo técnico y lo político obstaculiza las posibilidades de trabajo conjunto y de co-construcción de nuevos conocimientos que permitan la innovación y el mejoramiento de la producción a partir de estas formas laborales alternativas a la gestión de la empresa capitalista.

10 Mantenemos el uso del masculino, tal como lo expresaban las personas con las que conversamos de manera informal, observamos en reuniones y entrevistamos durante el trabajo de campo.

11 Desde una perspectiva de género podría interpretarse la discusión como un intento de colocar en un lugar subordinado a la mujer-técnica en un ámbito predominantemente político y masculino.. 
Esta división entre instancias técnicas y políticas estructura también el proceso de implementación de los programas del Ministerio de Desarrollo Social. Como mencionamos en la presentación del enfoque teórico-metodológico, la tarea de seguir a les actores a fin de evitar imponer un orden por anticipado y dejar que "desplieguen toda la gama de controversias en las que están inmersos" (Latour, 2008:42), nos llevó a acompañar a les integrantes de la Cooperativa La Huella en la gestión de recursos provistos por los programas de promoción del trabajo asociativo y autogestionado. En dicha situación observamos que el acceso a la solicitud de este implicó el paso por dos instancias diferenciadas. La primera fue el "acuerdo político" entre la Organización de la que participa La Huella, que permitió acercar a la cooperativa al Programa para que pudieran iniciar el trámite de solicitud del subsidio. Luego y de forma paralela a esta instancia, se realizó una evaluación "técnica", a fin de considerar la viabilidad del proyecto productivo y el cumplimiento de los requisitos formales del programa.

De este modo, el "acuerdo político" facilitaba un primer acceso a la obtención del subsidio, pero sólo en la medida en que los otros requisitos se cumplieran. Este acuerdo, tampoco contemplaba cómo iban a hacer los integrantes de La Huella para armar el proyecto y plasmarlo en el formulario estandarizado que era un requisito para acceder al programa, conseguir los tres presupuestos por cada recurso material y cumplir con los aspectos formales de elegibilidad, como tener personería jurídica, presentar los balances contables, el acta constitutiva de la cooperativa y tener los cambios de autoridades al día, entre otros.

Estas instancias de la gestión de la política social fueron llevados a cabo por distintes agentes: el "acuerdo político" entre el Estado Nacional (representado por funcionaries del Ministerio de Desarrollo Social) y la Organización (representada por sus referentes) y luego, la resolución de cuestiones "técnicas" para la elaboración del proyecto, de las que se ocuparon les llamades “asistentes técnicos” del Ministerio y les integrantes de la cooperativa que serían destinataries de la política social.

El "acuerdo político" estipulaba el monto máximo del subsidio que se otorgaría y los objetivos y lineamientos normativos del programa delimitaban qué tipo de actividades y qué recursos materiales podrían 
ser financiados. El atributo político en el caso de este acuerdo entre la Organización y el Ministerio parece aludir a una relación en donde se disputan intereses, tales como las condiciones de acceso a los programas y la cuantía de los recursos que podrán ser solicitados. Aquí la relación con funcionaries de distintos niveles gubernamentales forma parte del capital social de las organizaciones y cooperativas y se constituye en un recurso fundamental para visibilizar y plantear sus demandas colectivamente al Estado. Luego de esta primera instancia, les integrantes de La Huella comenzaron a armar el proyecto necesario para solicitar el financiamiento. En este proceso colaboraron algunas profesionales allegadas a la Organización ${ }^{12}$.

El armado, la búsqueda de los presupuestos y el cumplimiento de los requerimientos formales que estipulaba el programa, llevaron más de dos meses. Cuando estuvo todo listo, se presentó el proyecto y pasaron casi tres semanas sin novedades. El presidente de la Cooperativa llamó al Ministerio para averiguar qué sucedía, si habían comenzado a evaluar la solicitud, entonces uno de los asistentes técnicos del Ministerio explicó que estaba mal hecho el anexo 3 del formulario, que era un comprobante que debía tener los datos de la cuenta bancaria de la cooperativa. Además, le solicitó que rehicieran una de las actas, en la que se dejaba constancia de la decisión de solicitud del subsidio. Luego de esta conversación telefónica, el presidente de la cooperativa realizó las gestiones correspondientes y cuando consiguió todo lo que faltaba, volvió a presentarlo en el Ministerio. El mismo empleado con el que se había comunicado por teléfono recibió la documentación y dijo que era correcta, pero que aún faltaba modificar algunos aspectos de la formulación del proyecto (esto no había sido mencionado en la charla telefónica previa). Explicó que "lo que hay que cambiar son todas pavadas, detalles" pero después si eso no se corrige "lo pueden rebotar" en otra sección ${ }^{13}$ (Asistente técnico del Ministerio de Desarrollo Social de la Nación, 15 de noviembre de 2009). Por eso él tenía

12 Entre ellas la contadora que mencionamos antes. También la autora colaboró con la escritura del proyecto y su adaptación al formato de los formularios requeridos.

13 La evaluación de los proyectos se realiza en varias instancias (técnica, legal, administrativa, etc.) El proyecto es evaluado sucesivamente por las distintas áreas. Este proceso puede llevar varios meses. 
que fijarse minuciosamente en todas las $\operatorname{cosas}^{14}$. Finalmente, sugirió que se hicieran cuadros para organizar más claramente la propuesta y dijo que luego de que lo corrigieran y reorganizaran se lo enviaran por mail para que él pudiera chequearlo antes de que hicieran la entrega de los formularios definitivos en papel.

Todo este recorrido posterior al "acuerdo político" requirió tiempo y esfuerzo, el formulario era extenso y difícil de completar para personas que no tuvieran conocimientos específicos sobre el tema, más aún en el caso de les asociades de La Huella cuyo nivel de escolaridad formal era bajo y varies de elles, de nacionalidad paraguaya, hablaban principalmente en guaraní. Si el "acuerdo político" fue un primer paso para la solicitud del subsidio, la dificultad para completar formularios o cumplir con los trámites y requisitos, dificultaron y demoraron el acceso a los recursos que disponen estas políticas.

\section{Reflexiones Finales}

A partir del análisis de la experiencia de la cooperativa La Huella pudimos observar el modo en que las representaciones y valoraciones sobre lo técnico y lo político se ponen en juego en las prácticas cotidianas en distintos ámbitos y contextos. Los diferentes modos de entender estas cuestiones generan tensiones en el proceso de implementación de las políticas de promoción del trabajo asociativo y autogestionado y en la concreción de las metas que les distintes actores (personas, organizaciones, cooperativas y agencias estatales) se proponen a partir de su participación en los programas. Estas representaciones moldean también los límites y las condiciones de la participación, la distribución de tareas y responsabilidades e intervienen en las formas de acceso a los recursos que ofrecen estas políticas sociales.

En la Organización de la que participa la cooperativa, lo "político" da sentido a las acciones que el colectivo despliega para la transformación social y del trabajo. Se contrapone a lo "técnico" asociado, por un lado, a las

14 Una de las modificaciones era que había que quitarle el encabezado que tenía el formulario que les habían dado desde el Ministerio meses antes para que completaran el proyecto. El cambio en el formato del formulario se había decidido hacía poco, porque "a un secretario se le ocurrió que ahora va sin encabezado" (Asistente técnico del Ministerio de Desarrollo Social de la Nación, 15 de noviembre de 2009). 
exigencias de la intervención estatal que les son impuestas como obligaciones técnico-burocráticas y, por otro, a las decisiones de carácter individual de técniques que son excluidas del nosotres que conforma el colectivo. Así como en otros ámbitos, contextos e ideologías, se afirma la supremacía de la racionalidad técnica para la toma de decisiones, entendida como un saber pretendidamente objetivo, neutral y despolitizado que garantiza la eficacia y la eficiencia ${ }^{15}$, la construcción de lo político en el caso estudiado puede ser utilizada como una estrategia para desacreditar acciones y posiciones "técnicas". No obstante, al interior de la Organización estos sentidos son disputados. A partir de la experiencia profesional desde el Trabajo Social, esos mismos requisitos "técnicos" pueden ser entendidos y puestos en práctica como estrategias de apoyo a la autogestión, que al ser ejercidos por les integrantes de la Organización fortalecen el colectivo. Al mismo tiempo, el apoyo técnico para la contabilidad o para la elaboración de los proyectos necesarios para acceder al financiamiento que ofrecen los programas, constituye un recurso indispensable para lograr las metas que se proponen les cooperativistes. Sobre esta cuestión, podemos retomar la propuesta de Coraggio y Arancibia (2014) de asumir el Trabajo Social como parte de una práctica colectiva que recupere la economía en su intervención a fin de fortalecer procesos de transformación social, el acceso y la ampliación de derechos a partir de acciones tendientes a promover la autonomía de las personas y los grupos. Desde la perspectiva de la Economía Social que sostienen, esa autonomía no debe pensarse exclusivamente respecto de la asistencia o del apoyo estatal, sino también, en relación con las distintas formas de explotación del trabajo ajeno.

Los conflictos que pudimos observar a partir del trabajo de campo realizado se inscriben en una discusión más amplia acerca de los saberes y su reconocimiento. La innovación en las organizaciones y la construcción de nuevas formas de trabajo autogestionadas requiere del diálogo de distintos saberes, prácticas y experiencias técnicas y políticas, de la integración de saberes populares e informales (muchas veces invisibilizados y escasamente reconocidos) con conocimientos formalizados y especializados. Si como mostramos, la concepción dicotómica de lo técnico y lo político obstaculiza

15 Por ejemplo, en la concepción neoliberal del Estado y su visión tecnocrática de la política social. 
esas posibilidades, es necesario pensar cuáles son las condiciones que favorecerían esa articulación y esos diálogos necesarios. ¿Qué aporte específico podemos hacer desde un Trabajo Social comprometido con los procesos de organización colectiva para el reconocimiento de estas formas de trabajo "no clásicas"? (De la Garza Toledo, 2005).

Como mostramos, esta división estructuraba también la implementación de los programas del Ministerio de Desarrollo Social, ¿cuál es el rol que el Trabajo Social puede tener para facilitar el acceso a las políticas, junto al necesario registro, control y evaluación en la gestión de los recursos públicos? ¿Con qué herramientas teórico-metodológicas y técnico-operativas contamos para abrir un diálogo y tender puentes entre estas distintas lógicas de acción, representaciones y prácticas que se despliegan en la escala cotidiana de las políticas sociales? El conjunto de tareas y esfuerzos necesarios para acceder a los programas, no pueden ser entendidos solamente como trabas burocráticas ni pensarse como requisitos naturales. La experiencia de gestión de recursos estatales implica "salir" de la propia rutina y del universo de sentido de cada agente. Estos encuentros entre la política estatal y les destinataries, implican diversos aprendizajes que deben darse en dos direcciones. Desde les destinataries, se requiere un conocimiento acerca de las instituciones, sus pautas y reglas formales e informales de funcionamiento. Desde les agentes estatales, es necesario realizar un trabajo consciente para traducir esas reglas y acompañar los procesos, a fin de hacer accesibles y efectivos los derechos reconocidos por las políticas. El Trabajo Social tiene un rol central en esta tarea. De allí el desafío de problematizar individual y colectivamente lo que sucede en estos espacios e interacciones que se dan en la vida diaria de las instituciones públicas y en las organizaciones sociales. Como mostramos a partir del análisis realizado, estas instancias, habitualmente naturalizadas, son, sin embargo, las que hacen posible el acceso (o lo obstaculizan) y dan sentido a las políticas sociales.

\section{Referencias bibliográficas}

Aguilar Villanueva, Luis (1993). Problemas públicos y agenda de gobierno. Ciudad de México, México, Miguel Ángel Porrúa.

Aquin, Nora (2002). Nuevos escenarios y práctica profesional: una mirada crítica desde el Trabajo social. Buenos Aires, Argentina, Espacio. 
Bourdieu, Pierre y Wacquant, Loic (1995). Respuestas. Por una antropología reflexiva. Ciudad de México, México, Grijalbo.

Coraggio, José Luis y Arancibia, Inés (2014). Recuperando la economía: entre la cuestión social y la intervención social. Cuadernos de Trabajo Social, 27(1), 211-221. DOI: https:// doi.org/10.5209/rev CUTS.2014.v27.n1.40153

Danani, Claudia (1996). "La política social como campo de estudio y la noción de población-objeto". En S. Hintze (Ed.) Políticas Sociales. Contribuciones al debate teórico-metodológico (21-38). Buenos Aires, Argentina, EUDEBA-CEA.

De la Garza Toledo, Enrique (2005). Sindicatos y Nuevos Movimientos Sociales en América Latina. Buenos Aires, Argentina, CLACSO.

Grassi, Estela (2014). "Regímenes universalistas, derechos e igualdad. La escala cotidiana de las políticas sociales". En A, Arias, A, B. García Godoy, R. Manes (Ed.) Debates en torno a la construcción de institucionalidad (51-56). Buenos Aires, Argentina, UBA-Sociales Espacio Editorial.

Guber, Rosana (2009). El salvaje metropolitano. Reconstrucción del conocimiento social en el trabajo de campo. Buenos Aires, Argentina, Paidós.

Hintze, Susana y Deux Marzi, María Victoria (2007). La relación estado-sociedad en las políticas públicas de promoción de la Economía Social y Solidaria. V Jornadas Internacionales de Estado y Sociedad "Estado y sociedad en la búsqueda de nuevos roles", Facultad de Ciencias Económicas de la Universidad de Buenos Aires, CEDES, CONICET y ANPCyT, Buenos Aires, Argentina.

Hopp, Malena (2021). El trabajo: ¿medio de integración o recurso de la asistencia?. Buenos Aires, Argentina, Teseo.

Hopp, Malena (2015). Identidades laborales de destinatarios del Programa Ingreso Social con Trabajo "Argentina Trabaja". Trabajo y Sociedad, (24), 207-223. Recuperado de: https://www.unse.edu.ar/trabajoysociedad $/ 24 \% 20 \mathrm{Hopp} \% 20$ Malena $\% 20$ Identidades $\% 20$ Laborales.pdf

Latour, Bruno (2008). Reensamblar lo social. Una introducción a la teoría del actor-red. Buenos Aires, Argentina, Manantial.

Litman, Leila (2017). Producir desde la incomodidad Una economía moral del trabajo autogestionado (Tesis doctoral). Facultad de Filosofía y Letras de la Universidad de Buenos Aires, Buenos Aires, Argentina. Recuperado de: http://repositorio.filo.uba.ar/bitstream/handle/filodigital/6007/uba_ffyl t 2017 8282.pdf? sequence=1\&isAllowed=y

Minteguiaga, Analía (2003). El proceso de implementación del tercer ciclo de la educación general básica en el conurbano bonaerense, en el contexto de la reforma educativa, El caso de tres escuelas del Partido de Morón. (Tesis de Maestría) Facultad de Ciencias Sociales de la Universidad de Buenos Aires, Buenos Aires, Argentina.

Poole, Deborah (2012). Corriendo riesgos: normas, ley y participación en el Estado neoliberal, Anthropologica, (30), 83-100. Recuperado de: http://www.scielo.org.pe/pdf/anthro/ v30n30/a05v30n30.pdf

Penna, Maura (1992). O que faz ser nordestino. Identidades sociais, interesses e o 'escandalo'. Sao Paulo, Brasil, Cortez Editora.

Rockwell, Elsie (2009). La experiencia etnográfica: historia y cultura en los procesos educativos. Buenos Aires, Argentina, Paidós. 
Shore, Cris (2010). La antropología y el estudio de la política pública: reflexión sobre la "formulación" de las políticas, Antípoda, (10), 21-49. DOI: https://doi. org/10.7440/antipoda10.2010.03

Tamayo Sáez, Manuel (1997). El análisis de las políticas públicas. En R. Bañón y E. Carrillo (Ed.) La nueva Administración Pública (281-312). Madrid, España, Alianza.

Trouillot, Michel (2001). La antropología del Estado en la era de la globalización. Encuentros cercanos de tipo engañoso, Current Anthropology, 42(1), 125 138. Recuperado de: http://polsocytrabiigg.sociales.uba.ar/wp-content/uploads/ sites/152/2019/03/Trouillot-Antropolog\%C3\%ADa-del-Estado.pdf

Recibido: 07/07/2021

Aceptado: 12/10/2021 - 